\title{
Non-linear programming in shakedown analysis with plasticity and friction
}

A. Spagnoli, M. Terzano, J . R. Barber and Anders Klarbring

The self-archived version of this journal article is available at Linköping University Institutional Repository (DiVA):

http:// urn.kb.se/ resolve?urn=urn:nbn:se:liu:diva-139609

N.B.: When citing this work, cite the original publication.

Spagnoli, A., Terzano, M., Barber, J . R., Klarbring, A., (2017), Non-linear programming in shakedown analysis with plasticity and friction, J ournal of the mechanics and physics of solids, 104, 71-83. https:// doi.org/ 10.1016/j.jmps.2017.04.006

Original publication available at:

https:/ / doi.org/ 10.1016/j.jmps.2017.04.006

Copyright: Elsevier

http:// www.elsevier.com/

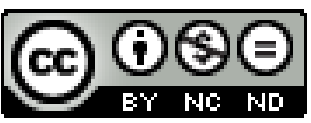




\title{
Non-linear programming in shakedown analysis with plasticity and friction
}

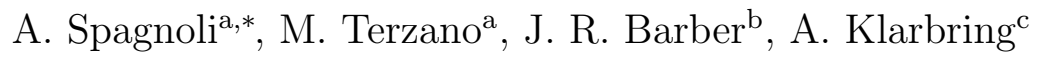 \\ ${ }^{a}$ Department of Engineering and Architecture, University of Parma, Viale Usberti \\ 181/A, 43124 Parma, Italy \\ ${ }^{b}$ Department of Mechanical Engineering, University of Michigan, Ann Arbor, MI \\ 48109-2125, USA \\ ${ }^{c}$ Department of Mechanical Engineering, Linköping University, S-581 83 Linköping, \\ Sweden
}

\begin{abstract}
Complete frictional contacts, when subjected to cyclic loading, may sometimes develop a favourable situation where slip ceases after a few cycles, an occurrence commonly known as frictional shakedown. Its resemblance to shakedown in plasticity has prompted scholars to apply direct methods, derived from the classical theorems of limit analysis, in order to assess a safe limit to the external loads applied on the system. In circumstances where zones of plastic deformation develop in the material (e.g., because of the large stress concentrations near the sharp edges of a complete contact), it is reasonable to expect an effect of mutual interaction of frictional slip and plastic strains on the load limit below which the global behaviour is non dissipative, i.e., both slip and plastic strains go to zero after some dissipative load cycles. In this paper, shakedown of general two-dimensional discrete systems, involving both friction and plasticity, is discussed and the shakedown limit load is calculated using a non-linear programming algorithm based on the static theorem of limit analysis. An illustrative example related to an elastic-plastic solid containing a frictional crack is provided.
\end{abstract}

Keywords: limit analysis, shakedown, complete contact, friction, non-linear programming

\footnotetext{
${ }^{*}$ Corresponding author. Tel.: +39-0521-905927; fax: +39-0521-905924; e-mail: spagnoli@unipr.it.
} 


\section{Introduction}

It is a common occurrence for structural systems to be exposed to a combination of loads of different nature, some of which vary in time, such as temperature or vibrations. An elastic-plastic system subjected to variable repeated actions may fail as a result of alternating plasticity, comprising equal plastic strains of opposite sign leading eventually to local material failure, or by ratcheting or incremental collapse, that is, a progressive accumulation of plastic strain leading to local failure ol loss of stability (König and Maier, 1981). Alternatively, it may happen that, after a certain time, plastic strains cease to develop further and the behaviour to the subsequent loading cycles is purely elastic: this is the condition of adaptation or shakedown.

An analogous behaviour is observed in systems which comprise elastic components in mutual frictional contact. When subjected to variable loads, microslip can occur along limited portions of the contact interfaces, which play the role of the plastic strains in the monolithic elastic-plastic system (Churchman et al., 2006). The effect of microslip on system performance results in the well known damage process of fretting fatigue, which reduces the service life of components (Nowell et al., 2006). It is therefore natural to investigate the safe condition, for which microslip ceases to develop after an initial transient stage and a state of permanent stick with non dissipative behaviour follows. This condition is commonly known as frictional shakedown. Numerical analyses of complete contacts, i.e., when the contact area is known a-priori and does not change in the presence of applied loads, sometimes predict that the contact shakes down (Churchman and Hills, 2006), that is, after the first few loading cycles, a residual state is developed that inhibits slip for all subsequent cycles.

Methods which provide tools for safety assessment of systems under variable loads, namely to establish a load limit under which the shakedown is guaranteed, are highly attractive. The so-called simplified or direct methods based on the shakedown theorems of limit analysis offer an advantageous approach, particularly if compared to more traditional incremental analyses (also termed step-by-step or marching analyses). Indeed, in practical problems we often do not know the precise loading history or initial conditions needed for step-by-step simulations, which in any case are generally computationally cumbersome.

In this paper we limit our attention to direct methods obtained from shakedown theorems and their implementation in mathematical program- 
ming. Originally developed in the framework of classical plasticity (Melan, 1936; Koiter, 1960), shakedown theorems have been extended to more general material models, i.e., to include non associated plasticity (Maier, 1969), geometric non linearities (Weichert, 1986), non-linear hardening (Pycko and Maier, 1995), Signorini's boundary conditions with and without friction (Telega, 1995), elastic-plastic damage materials (Polizzotto et al., 2002). An application of shakedown theorem to so-called bridged cracks has been presented in Spagnoli et al. (2013) to describe the dissipative condition of fibrereinforced beams. As for frictional shakedown, the evident similarity with shakedown in plasticity has prompted scholars to formulate a static shakedown theorem for frictional contacts, in the context of discrete (Klarbring et al., 2007) and continuum problems (Barber et al., 2008), with the limitation of normal-tangential uncoupling along the contact interface. Another contribution in this field came from considering the condition of shakedown in a system which comprises parts in frictional contacts and elastic-plastic material behaviour. The safe condition must be defined, as usual, by considering the lack of dissipation, which in this case refers to both plastic deformation and frictional slip (Klarbring et al., 2017).

The aim of this paper is to study the shakedown condition in a discrete system with standard material law, i.e., elastic-perfectly plastic with associated flow rule, and complete contact interfaces, upon which the behaviour is defined by Coulomb friction. A direct method, based on the static formulation of shakedown theorems, is developed in the framework of non-linear convex optimisation.

The outline of the paper is as follows. In $\S 2$ we present the formulation of the discrete contact problem. In $\S 3$, direct methods relying on mathematical programming are considered and the non-linear algorithm used is presented. Finally, in $\S 4$, the algorithm is applied to an illustrative two-dimensional example of an elastic-plastic solid containing a frictional crack, and the results are presented and discussed.

\section{Formulation}

We begin our analysis by considering a two-dimensional discrete system with a complete contact geometry, such as the one displayed in Figure 1. As mentioned earlier, the only requirement we introduce with this assumption is that the contact region does not change in size during the whole loading history. Several contact problems are therefore included, such as the inden- 


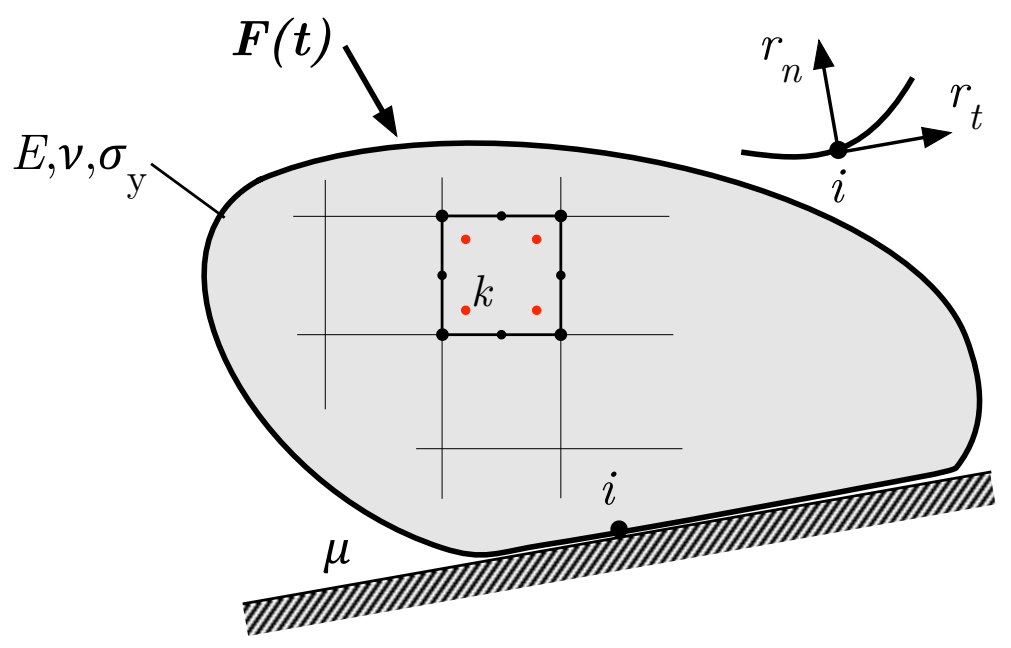

Figure 1. Schematic figure of an elastic-plastic system with parts in frictional contact. Discretised mesh and positive conventions on a generic contact node are also displayed.

tation of a half-space by a punch or the case of two elastic bodies separated along conforming surfaces. Let us assume an homogeneous elastic-perfectly plastic material with associated flow rule and Coulomb's law of friction along the interfaces.

The system is discretised, e.g. by means of the finite element method, in such a way that there are $M$ contact nodes and $K$ integration points. Then we can collect nodal displacements in a vector $\boldsymbol{u}$ which approximates the continuous displacement field and strains at integration points in a vector $\varepsilon$. Under the hypothesis of small displacements, we define a matrix $\boldsymbol{B}$ which connects the strains to the nodal displacements, so that we can write

$$
\varepsilon=B u
$$

On the contact interfaces we collect the displacements at each node, which are relative displacements in case of two-body contact, in a vector $\boldsymbol{w}$ connected to the vector $\boldsymbol{u}$ by means of a transformation matrix $\boldsymbol{C}$, such that

$$
\boldsymbol{w}=\boldsymbol{C u}
$$

At contact nodes we also collect the reaction forces in a vector $\boldsymbol{r}$. Contact displacements and forces may be decomposed in the components related to 
the local tangential and normal directions and ordered in vectors as follows

$$
\begin{gathered}
\boldsymbol{w}=\left[\boldsymbol{w}_{\boldsymbol{t}}^{T} \boldsymbol{w}_{\boldsymbol{n}}^{T}\right]^{T}=\left[w_{1 t}, \ldots, w_{M t} w_{1 n}, \ldots, w_{M n}\right]^{T} \\
\boldsymbol{r}=\left[\boldsymbol{r}_{\boldsymbol{t}}^{T} \boldsymbol{r}_{\boldsymbol{n}}^{T}\right]^{T}=\left[r_{1 t}, \ldots, r_{M t} r_{1 n}, \ldots, r_{M n}\right]^{T}
\end{gathered}
$$

The convention adopted considers the normal component of displacement $\boldsymbol{w}_{\boldsymbol{n}}$ positive when it opens a gap in the contact while the reaction force $\boldsymbol{r}_{\boldsymbol{n}}$ is positive when compressive, see Figure 1.

Using the transformation matrix $\boldsymbol{C}$, we can obtain a contact stiffness matrix $\boldsymbol{\kappa}$ from the global stiffness matrix of the system $\boldsymbol{K}$ as

$$
\boldsymbol{\kappa}^{-1}=\boldsymbol{C K}^{-1} \boldsymbol{C}^{T}
$$

where $\boldsymbol{\kappa}$ is a symmetric and positive semi-definite $2 M \times 2 M$ matrix, which may be singular if the system can perform rigid-body displacements along the contact surface. The global stiffness matrix is obtained from the straindisplacement matrix $\boldsymbol{B}$ as

$$
\boldsymbol{K}=\boldsymbol{B}^{T} \boldsymbol{E} \boldsymbol{D}
$$

where $\boldsymbol{E}$ is the elasticity matrix and $\boldsymbol{D}=\boldsymbol{V} \boldsymbol{B}$, with $\boldsymbol{V}$ being a diagonal matrix containing the volume associated to each integration point of finite elements. In order to make explicit normal-tangential coupling at the contact interface, we may decompose $\boldsymbol{\kappa}$ as

$$
\left[\begin{array}{cc}
\kappa_{t t} & \kappa_{n t}^{T} \\
\kappa_{\boldsymbol{n} t} & \kappa_{\boldsymbol{n} \boldsymbol{n}}
\end{array}\right]
$$

where the sub-matrices correspond to the following force-displacement relationships: tangential-tangential, $\boldsymbol{\kappa}_{t t}$; normal-tangential, $\boldsymbol{\kappa}_{n t}$; normal-normal, $\kappa_{n n}$.

\subsection{Linear elastic response}

We now consider the application of to apply external loads $\boldsymbol{F}$ to the system, assuming elastic behaviour and welded contact nodes. In this state the response of the system, denoted by index $E$, is linear elastic and the strain field is such that

$$
\varepsilon=e^{E}
$$

and, according to (2), the displacement field

$$
\mathbf{0}=\boldsymbol{C} \boldsymbol{u}^{E}
$$


Stresses are obtained from Hooke's law as

$$
\boldsymbol{\sigma}^{E}=\boldsymbol{E} \boldsymbol{e}^{E}
$$

In this state, the global equilibrium of the system is described by the following equation

$$
\boldsymbol{F}+\boldsymbol{C}^{T} \boldsymbol{r}^{E}=\boldsymbol{D}^{T} \boldsymbol{\sigma}^{E}
$$

where vector $\boldsymbol{r}^{E}$ represents the reaction forces that are generated at the contact nodes if relative displacements are constrained to be zero, i.e., if the interfaces are welded together.

In our analysis, we considered an external loading additively decomposed into time constant component $\boldsymbol{F}_{0}$ and a time varying cyclic component $\beta \overline{\boldsymbol{F}}(t)$, where $\beta$ is the so-called load multiplier. We may write

$$
\begin{aligned}
\boldsymbol{\sigma}^{E} & =\boldsymbol{\sigma}_{0}^{E}+\beta \overline{\boldsymbol{\sigma}}^{E}(t) \\
\boldsymbol{r}^{E} & =\boldsymbol{r}_{0}^{E}+\beta \overline{\boldsymbol{r}}^{E}(t)
\end{aligned}
$$

where $\boldsymbol{\sigma}_{0}^{E}$ and $\boldsymbol{r}_{0}^{E}$ are, respectively, stresses and contact forces corresponding to the time constant force $\boldsymbol{F}_{0}$, while $\overline{\boldsymbol{\sigma}}^{E}(t)$ and $\overline{\boldsymbol{r}}^{E}(t)$ are, respectively, stresses and contact forces related to the time varying force $\overline{\boldsymbol{F}}(t)$.

\subsection{The yield condition}

The yield condition marks the limit of the elastic domain and defines the plasticity behaviour of the material. In our analysis, we assume a standard material, i.e., a material with associated flow of plastic strains, and perfectly plastic behaviour. For such materials, the elastic domain is represented by a convex region in the space of the stress components $\sigma_{i j}$, delimited by a yield function $f$. With a conventional notation in the theory of plasticity we write

$$
\begin{aligned}
& f\left(\sigma_{i j}\right) \leq 0 \\
& \dot{p}_{i j}=\dot{\lambda} \frac{\partial f}{\partial \sigma_{i j}} \\
& f \dot{\lambda}=0
\end{aligned}
$$

where $\dot{\lambda}$ is a non-negative scalar quantity and $\dot{p}_{i j}$ is the time derivative of the plastic strain, that is, the plastic flow. Equation (14b) is also known as normality rule, since the vector representing the plastic flow at one material point is normal to the yield function, see e.g. the Von Mises yield condition in Figure 2a. 


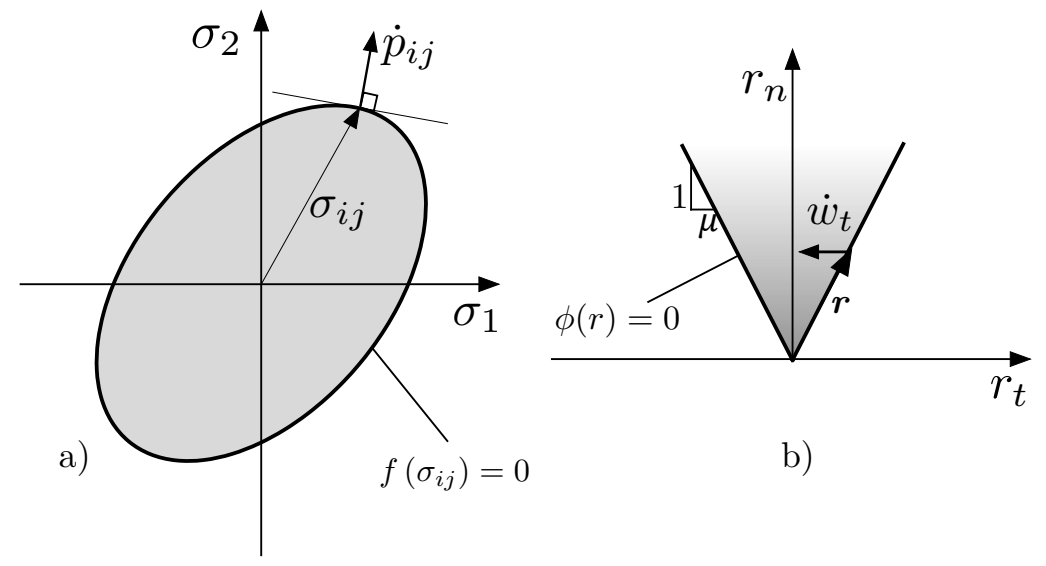

Figure 2. Limit conditions for two-dimensional systems. a) Von Mises yield condition with associated flow in the space of principal stresses. b) Coulomb slip function in the space of contact forces.

\subsection{The friction law}

Along the contact interfaces, the behaviour is defined by the Coulomb friction law, which can be formulated as a rigid-plastic constitutive law with a single parameter, the coefficient of friction $\mu$. Following the same formalism used to define the yield condition, the Coulomb friction law may be described by a slip function and a flow rule (Figure 2b)

$$
\begin{aligned}
& \phi(\boldsymbol{r})=\left|r_{t}\right|-\mu r_{n} \leq 0 \\
& \dot{w}_{t}=-\dot{\lambda} \frac{r_{t}}{\left|r_{t}\right|} \\
& \phi \dot{\lambda}=0
\end{aligned}
$$

where $\dot{\lambda}$ is a non-negative scalar quantity and $\dot{w}_{t}$ is the time derivative of the tangential contact displacement, that is, the slip flow. This law specifies when and how slip takes place: slip is zero when contact forces belong to an admissible set, the so called Coulomb cone of friction, and it otherwise opposes the shear force.

It is evident that the Coulomb friction law is non-associated, since the direction of slip flow is defined by the tangential component of the contact reaction, which is not generally normal to the slip function. More correctly, we can say that the normality rule is satisfied by the slip flow $\dot{w}_{t}$ and the 
tangential reaction $r_{t}$, but the set of admissible forces depends on the present value of $r_{n}$. This means that, whenever we can consider the normal reaction independent on slip, that is to say, when normal reaction is determined by the sole equilibrium with external loads, the normality rule applies (Michalowski and Mroz, 1978). This is exactly the case for uncoupled contacts where there is no normal-tangential coupling, meaning that contact normal reactions are not modified by tangential contact displacements.

\subsection{Residual state}

At a generic time instant of the loading history we can remove the external loads, $\boldsymbol{F}=\mathbf{0}$, keeping $\boldsymbol{w}$ and $\boldsymbol{p}$ fixed. Thus, a residual state, denoted by index $R$, will exist in the system, which is self-equilibrated and accounts for the permanent part of the deformation. Note that this residual state might not be the one caused by the applied loading history, but, when summed up with the elastic (time-varying) state, it will have to satisfy 'no flow' conditions of plasticity and friction. Moreover, we restrict our attention to complete contacts situations (no separation), for which all the nodes remain in contact during the whole loading history, i.e. $\boldsymbol{w}_{\boldsymbol{n}}=\mathbf{0}$.

The residual strain field of the system is defined as

$$
\varepsilon^{R}=e^{R}+\boldsymbol{p}
$$

where the total strain vector is the sum of a plastic component and an elastic residual component. Residual stresses can be obtained, as usual, from the generalized Hooke law

$$
\boldsymbol{\sigma}^{R}=\boldsymbol{E} \boldsymbol{e}^{R}=\boldsymbol{E}\left(\boldsymbol{B} \boldsymbol{u}^{R}-\boldsymbol{p}\right)
$$

where $\boldsymbol{u}^{R}$ is the vector of residual nodal displacements. The global equilibrium of the system is described by the equation

$$
\boldsymbol{C}^{T} \boldsymbol{r}^{R}=\boldsymbol{D}^{T} \boldsymbol{\sigma}^{R}
$$

Combining equations (2), (5), (6), (17) and (18) we are able to express the contact residual forces as a function of $\boldsymbol{p}$ and $\boldsymbol{w}$

$$
\boldsymbol{r}^{R}=\boldsymbol{r}^{R}(\boldsymbol{p})+\boldsymbol{r}^{R}(\boldsymbol{w})=-\boldsymbol{\kappa} \boldsymbol{C} \boldsymbol{K}^{-1} \boldsymbol{D}^{T} \boldsymbol{E} \boldsymbol{p}+\boldsymbol{\kappa} \boldsymbol{w}
$$

Moreover, introducing (17) and (19) in the equilibrium equation (18), one may express the residual displacement $\boldsymbol{u}^{R}$ as a linear function of plastic strains and contact displacements

$$
\boldsymbol{u}^{R}=\boldsymbol{A}_{p} \boldsymbol{p}+\boldsymbol{A}_{w} \boldsymbol{w}
$$


where

$$
\begin{aligned}
& \boldsymbol{A}_{p}=\boldsymbol{K}^{-1}\left(\boldsymbol{D}^{T} \boldsymbol{E}-\boldsymbol{C}^{T} \boldsymbol{\kappa} \boldsymbol{C} \boldsymbol{K}^{-1} \boldsymbol{D}^{T} \boldsymbol{E}\right) \\
& \boldsymbol{A}_{w}=\boldsymbol{K}^{-1} \boldsymbol{C}^{T} \boldsymbol{\kappa}
\end{aligned}
$$

Then the final form of the residual stresses $\boldsymbol{\sigma}^{R}$ in (17) is the following

$$
\boldsymbol{\sigma}^{R}=\left(\boldsymbol{E} \boldsymbol{B} \boldsymbol{A}_{p}-\boldsymbol{E}\right) \boldsymbol{p}+\boldsymbol{E} \boldsymbol{B} \boldsymbol{A}_{w} \boldsymbol{w}
$$

If the contact is uncoupled, the contact stiffness matrix is block diagonal, since the normal-tangential component $\boldsymbol{\kappa}_{n t}$ in (7) is null. Therefore, from (19), the part of the residual contact reaction which accounts for relative displacements is simplified as follows

$$
\boldsymbol{r}^{R}(\boldsymbol{w})=\left[\boldsymbol{r}_{t}^{R^{T}} \boldsymbol{r}_{n}^{R^{T}}\right]^{T}=\left[\boldsymbol{w}_{\boldsymbol{t}}^{\boldsymbol{T}} \boldsymbol{\kappa}_{\boldsymbol{t} \boldsymbol{t}} \mathbf{0}^{T}\right]^{T}
$$

The same argument can be used for $\boldsymbol{r}^{R}(\boldsymbol{p})$. This is a fundamental assertion: when there is no normal-tangential coupling, the residual state of the system along the contact interfaces does not have an influence on the distribution of the normal contact forces, which will therefore be purely linear elastic.

Reintroducing the external loads, we obtain a linear elastic state which can simply be added to the residual state. Stresses at integration points and forces at contact nodes are therefore expressed as

$$
\begin{gathered}
\boldsymbol{\sigma}=\boldsymbol{\sigma}^{E}+\boldsymbol{\sigma}^{R}=\boldsymbol{\sigma}_{0}^{E}+\beta \overline{\boldsymbol{\sigma}}^{E}(t)+\left(\boldsymbol{E} \boldsymbol{B} \boldsymbol{A}_{p}-\boldsymbol{E}\right) \boldsymbol{p}+\boldsymbol{E} \boldsymbol{B} \boldsymbol{A}_{w} \boldsymbol{w} \\
\boldsymbol{r}=\boldsymbol{r}^{E}+\boldsymbol{r}^{R}=\boldsymbol{r}_{0}^{E}+\beta \overline{\boldsymbol{r}}^{E}(t)-\boldsymbol{\kappa} \boldsymbol{C} \boldsymbol{K}^{-1} \boldsymbol{D}^{T} \boldsymbol{E} \boldsymbol{p}+\boldsymbol{\kappa} \boldsymbol{w}
\end{gathered}
$$

\section{Shakedown in mathematical programming}

Direct methods are applied to the analysis of variable repeated loads acting on elastic-plastic systems, with the main purpose of establishing a safe condition for the shakedown, without following a step-by-step procedure.

Let us consider the external load expressed as $\boldsymbol{F}(t)=\boldsymbol{F}_{0}+\beta \overline{\boldsymbol{F}}(t)$. We assume the cyclic load to be enclosed by a convex domain $\Omega$, which is a hyperpolyhedron delimited by a linear combinations of load vertices $F_{j}(j=1, \ldots, J)$ in the most general case. It is assumed that if shakedown occurs under any load vertices, then it will occur under the whole load domain $\Omega$ (König, 
1987): this means that we can replace any cycle of loading by the cycle passing through the vertices and use the discrete values $t_{j}(j=1, \ldots, J)$ of the functions of $t$. The load multiplier $\beta$ is sufficient to describe the size of this domain and we can use direct methods to find a safety factor, i.e., the shakedown load multiplier $\beta_{S}$, which ensures that, for any $\beta \leq \beta_{S}$, the system shakes down, while for $\beta>\beta_{S}$ it does not.

\subsection{Non-linear programming for associated plasticity}

Most of the used direct methods in associated plasticity rest on Melan's static shakedown theorem (Melan, 1936) and its formulation as a mathematical programming problem. Melan's theorem affirms that a sufficient condition for the shakedown is the existence of a residual state $\boldsymbol{\sigma}^{R}$ which, added to the time varying elastic response $\boldsymbol{\sigma}^{E}(t)$, does not violate the yield condition.

For the considered load domain, at each integration point $k$ a statically admissible stress state $\boldsymbol{\sigma}_{k}=\boldsymbol{\sigma}_{k 0}^{E}+\beta \overline{\boldsymbol{\sigma}}_{k}^{E}(t)+\boldsymbol{\sigma}_{k}^{R}$ is such if it satisfies the yield condition $f_{k}\left(\boldsymbol{\sigma}_{k}\right) \leq 0$. Then the shakedown load multiplier $\beta_{S}$ is obtained with a mathematical non-linear programming problem by maximizing the statically admissible multiplier $\beta$

$$
\begin{aligned}
& \beta_{S}=\max _{\beta, \boldsymbol{\sigma}^{R}} \beta \quad \text { subject to : } \\
& \text { (I) } \quad f_{k}\left(\boldsymbol{\sigma}_{k 0}^{E}+\beta \overline{\boldsymbol{\sigma}}_{k}^{E}\left(t_{j}\right)+\boldsymbol{\sigma}_{k}^{R}\right) \leq 0 \quad k=1, \ldots, K, \quad j=1, \ldots, J \\
& \text { (II) } \boldsymbol{D}^{T} \boldsymbol{\sigma}^{R}=\mathbf{0} \\
& \text { (III) } \beta \geq 0
\end{aligned}
$$

where we considered the jth vertex of the load domain at which the time functions are computed. Constraint (II) in (26) is the mathematical statement of the condition of self-equilibrium of the residual stresses while constraint (III) is the requirement of non negativity of the load multiplier. Such a problem, for a convex quadratic yield function, leads to a numerical optimisation involving a linear objective function under linear and quadratic constraints (known as QCLP, i.e., quadratically constrained linear problem).

A computational advantage in this type of optimisations may be obtained by using a piecewise linearisation of the yield function (Maier, 1969) and considering the mathematical dual problem, which reduces the number of constraints dramatically (Corradi and Zavelani, 1974). However, this approach 
may prove to be inefficient for large problems and recently increased efficiency has been reached by transforming the quadratic constraints into conic constraints (see, for instance, Andersen et al., 2003). Different approaches for calculating the shakedown limit are also possible, for instance Pycko and Mroz (1992) proposed an alternative min-max procedure while others ( $\mathrm{Li}$ and $\mathrm{Yu}, 2006)$ used an algorithm derived from Koiter's kinematic theorem.

We note that the mathematical problem in (26) can also be applied to a system involving frictionless contact boundary conditions with an elasticperfectly plastic material, with some modifications to the constraints of the optimisation as follows

$$
\begin{aligned}
& \beta_{S}=\max _{\beta, \boldsymbol{\sigma}^{R}, \boldsymbol{r}^{R}} \beta \quad \text { subject to : } \\
& \quad(I) \quad f_{k}\left(\boldsymbol{\sigma}_{k 0}^{E}+\beta \overline{\boldsymbol{\sigma}}_{k}^{E}\left(t_{j}\right)+\boldsymbol{\sigma}_{k}^{R}\right) \leq 0 \quad k=1, \ldots, K, \quad j=1, \ldots, J \\
& (I I) \quad \boldsymbol{C}^{T} \boldsymbol{r}^{R}=\boldsymbol{D}^{T} \boldsymbol{\sigma}^{R} \\
& (I I I) \quad \beta \geq 0 \\
& \text { (IV) } \boldsymbol{r}_{t}=\mathbf{0} \\
& \text { (V) } \quad \boldsymbol{r}_{n} \geq \mathbf{0}
\end{aligned}
$$

\subsection{Linear programming for friction}

Classical shakedown theorems require the flow law to be associated. In frictional systems, as was noted in $§ 2.3$, this condition is not satisfied, except in the case of uncoupled contacts. However, necessary and sufficient conditions for shakedown with non-associated plasticity, which in principle can be generalized to friction problems, were given by Maier (1969). Unfortunately, the lower bound theorem, which rests on the assumption of the existence of a reduced elastic domain with associated flow, is not helpful in the case of friction. Indeed, it reduces to the half line of positive normal contact forces with zero tangential forces and the calculated lower bound for the load is null (Björkman and Klarbring, 1987).

As shown by Björkman and Klarbring (1987), a different approach which yields an upper bound to the frictional shakedown load can be adopted. This approach derives from Maier's necessary condition for shakedown with non-associated flow, which is stated in the following terms: if shakedown occurs, a time-independent residual contact force $\boldsymbol{r}^{R}$ exists such that the superimposition of this state on the elastic contact response $\boldsymbol{r}^{E}$ at no point of the contact surface violates the slip criterion. 
For the considered load domain, at each contact node $i$ the total contact force $\boldsymbol{r}_{i}=\boldsymbol{r}_{i 0}^{E}+\beta \overline{\boldsymbol{r}}_{i}^{E}(t)+\boldsymbol{r}_{i}^{R}$ must respect the slip condition $\phi_{i}\left(\boldsymbol{r}_{i}\right) \leq 0$. Then the shakedown load multiplier $\beta_{S}$ is obtained with a mathematical programming problem by maximizing the load multiplier $\beta$

$$
\begin{aligned}
& \beta_{S}=\max _{\beta, \boldsymbol{r}^{R}} \beta \quad \text { subject to : } \\
& \text { (I) } \quad \phi_{i}\left(\boldsymbol{r}_{i 0}^{E}+\beta \overline{\boldsymbol{r}}_{i}^{E}\left(t_{j}\right)+\boldsymbol{r}_{i}^{R}\right) \leq 0 \quad i=1, \ldots, M, \quad j=1, \ldots, J \\
& \text { (II) } \beta \geq 0 \\
& \text { (III) } \quad \boldsymbol{r}_{n} \geq \mathbf{0}
\end{aligned}
$$

where we considered the jth vertex of the load domain at which the time functions are computed. Constraint (I) in (28) expresses the slip condition while constraint (III) puts in mathematical terms the condition that contact forces cannot be tensile.

The choice of an appropriate slip function $\phi$ determines the nature of the constraints. For Coulomb's law of friction, the slip function is the one defined in (15a), and in this case the previous problem leads to a numerical optimisation involving a linear objective function under linear constraints (known as LP, i.e., linear problem). The complete formulation of this algorithm is given in Björkman and Klarbring (1987), whose article should be consulted for an in-depth explanation. The slip condition at each contact node $i$ is written as

$$
\phi_{i}^{\alpha}=\boldsymbol{N}_{i}^{\alpha T} \boldsymbol{r}_{i} \leq 0
$$

where $\boldsymbol{N}_{i}^{\alpha}$ is the unit vector normal to the slip function for backward slip $(\alpha=1)$ and forward $\operatorname{slip}(\alpha=2)$. The time varying component of the elastic contact forces $\overline{\boldsymbol{r}}_{i}^{E}\left(t_{j}\right)$ can be replaced by considering, for each slip function, the maximum with respect to the vertices of the load domain of the projection of $\overline{\boldsymbol{r}}_{i}^{E}\left(t_{j}\right)$ on the normal vector, that is

$$
M_{i}^{\alpha}=\max _{j}\left(\boldsymbol{N}_{i}^{\alpha T} \overline{\boldsymbol{r}}_{i}^{E}\left(t_{j}\right)\right), \quad j=1, \ldots, J
$$

The scalar quantities $M_{i}^{\alpha}$ can be arranged in the vector $\boldsymbol{M}$ pertaining to the whole contact interface and the unit vector $N_{i}^{\alpha}$ can be assembled in a block diagonal matrix $\boldsymbol{N}$. Using equations (25) and (30) in (29), the slip condition $(I)$ in (28) is rewritten as

$$
\beta \boldsymbol{M}+\boldsymbol{r}^{R} \leq-\boldsymbol{N}^{T} \boldsymbol{r}_{0}^{E}
$$


Recently, Ahn (2015) efficiently applied linear programming in the calculation of the upper shakedown limit of an elastic block pressed against a frictional rigid plane. Flicek et al. (2015) proposed a different approach to the shakedown limit calculation, by using a min-max algorithm and expressing the safe condition for shakedown in terms of the minimum coefficient of friction below shakedown is impossible.

\subsection{Non linear programming algorithm for plasticity and friction}

When friction and plasticity are considered at the same time, a new definition of the safe condition for the shakedown has to be introduced, which involves both the shakedown of plastic strains and frictional slip. We here recall that the shakedown condition in a frictional elastic-plastic system is one that ensures

(i) $\dot{\boldsymbol{p}}=\mathbf{0}$. Plastic strain ceases after the initial transient stage and the response is purely elastic;

(ii) $\dot{\boldsymbol{w}}_{\boldsymbol{t}}=\mathbf{0}$. Frictional slip ceases after the initial transient stage and a state of stick is restored along the contact interface.

The condition of shakedown is ensured by the existence of a residual state $\left(\boldsymbol{\sigma}^{R}, \boldsymbol{r}^{R}\right)$ such that, for all times, superimposed on the linear elastic response due to the external loads, satisfies the yield condition $f_{k}\left(\boldsymbol{\sigma}_{k}\right) \leq 0$ in the stress space and the slip condition $\phi_{i}\left(\boldsymbol{r}_{i}\right) \leq 0$ in the space of contact reaction forces. It has been proved that the existence of such a residual state is also a sufficient condition for shakedown (Klarbring et al., 2017) in the case of uncoupled contacts.

For the considered load domain we may express the total stress state at each integration point $k$ as $\boldsymbol{\sigma}_{k}=\boldsymbol{\sigma}_{k 0}^{E}+\beta \overline{\boldsymbol{\sigma}}_{k}^{E}(t)+\boldsymbol{\sigma}_{k}^{R}$ and the total contact force at each contact node $i$ as $\boldsymbol{r}_{i}=\boldsymbol{r}_{i 0}^{E}+\overline{\boldsymbol{r}}_{i}^{E}(t)+\boldsymbol{r}_{i}^{R}$. The shakedown load multiplier $\beta_{S}$ is obtained with a mathematical non-linear programming problem by maximizing the load multiplier $\beta$, provided that both the limit conditions are satisfied. Using equations (19) and (22) we express the residual state as a function of plastic strains $\boldsymbol{p}$ and of contact displacements $\boldsymbol{w}$, which therefore are the variables of the programming problem, together with the load parameter $\beta$. We obtain 
$\beta_{S}=\max _{\beta, \boldsymbol{w}, \boldsymbol{p}} \beta \quad$ subject to :

(I) $f_{k}\left(\boldsymbol{\sigma}_{k 0}^{E}+\beta \overline{\boldsymbol{\sigma}}_{k}^{E}\left(t_{j}\right)+\left(\boldsymbol{E} \boldsymbol{B} \boldsymbol{A}_{p}-\boldsymbol{E}\right) \boldsymbol{p}+\boldsymbol{E} \boldsymbol{B} \boldsymbol{A}_{w} \boldsymbol{w}\right) \leq 0 \quad k=1, \ldots, K$,

$j=1, \ldots, J$

(II) $\phi_{i}\left(\boldsymbol{r}_{i 0}^{E}+\beta \overline{\boldsymbol{r}}_{i}^{E}\left(t_{j}\right)-\boldsymbol{\kappa} \boldsymbol{C} \boldsymbol{K}^{-1} \boldsymbol{D}^{T} \boldsymbol{E} \boldsymbol{p}+\boldsymbol{\kappa} \boldsymbol{w}\right) \leq 0$

$i=1, \ldots, M$

$j=1, \ldots, J$

(III) $\beta \geq 0$

(IV) $\boldsymbol{w}_{n}=\mathbf{0}$

(V) $\boldsymbol{r}_{n}(\boldsymbol{w}, \boldsymbol{p}) \geq \mathbf{0}$

Constraint (I) expresses the yield condition at each integration point of the discrete system. In case of the Von Mises yield function it is quadratic and takes the following form

$$
f_{k}\left(\boldsymbol{\sigma}_{k}\right)=\frac{1}{2} \boldsymbol{\sigma}_{k}^{T} \boldsymbol{H} \boldsymbol{\sigma}_{k}-\sigma_{y}^{2} \leq 0
$$

where $\boldsymbol{H}$ is a symmetric yield matrix, $\boldsymbol{\sigma}_{k}$ is the vector of stresses $\sigma_{i j}$ at the kth integration point and $\sigma_{y}$ is the uniaxial yield strength of the material. In plane stress conditions, the vector $\boldsymbol{\sigma}_{k}$ contains the stresses $\sigma_{11}, \sigma_{22}, \sigma_{12}$ at each integration point and $\boldsymbol{H}$ is expressed as follows

$$
\mathbf{H}=\left[\begin{array}{ccc}
2 & -1 & 0 \\
-1 & 2 & 0 \\
0 & 0 & 6
\end{array}\right]
$$

Since the ordered vector of variables in the programming problem is $\boldsymbol{x}=$ $\left[\beta \boldsymbol{p}^{T} \boldsymbol{w}^{T}\right]^{T}$, we need to rewrite the yield condition as follows in order to maintain the quadratic form

$$
\frac{1}{2} \boldsymbol{x}^{T} \boldsymbol{Q}_{k}^{j} \boldsymbol{x}+\boldsymbol{q}_{k}^{j T} \boldsymbol{x}+d_{k} \leq 0
$$

where $\boldsymbol{Q}_{k}^{j}$ is the positive semi-definite matrix of the quadratic terms, $\boldsymbol{q}_{k}^{j}$ is the vector of the linear terms, both related to the jth vertex of the load domain, 
and $d_{k}$ is the known term. These terms have the following expressions

$$
\begin{aligned}
\boldsymbol{Q}_{k}^{j}= & {\left[\begin{array}{ccc}
\overline{\boldsymbol{\sigma}}_{k}^{E}\left(t_{j}\right)^{T} \boldsymbol{H} \overline{\boldsymbol{\sigma}}_{k}^{E}\left(t_{j}\right) & \text { sym } \\
\boldsymbol{Q}_{p}^{T} \boldsymbol{H} \overline{\boldsymbol{\sigma}}_{k}^{E}\left(t_{j}\right) & \boldsymbol{Q}_{p}^{T} \boldsymbol{H} \boldsymbol{Q}_{p} & \\
\boldsymbol{Q}_{w}^{T} \boldsymbol{H} \overline{\boldsymbol{\sigma}}_{k}^{E}\left(t_{j}\right) & \boldsymbol{Q}_{w}^{T} \boldsymbol{H} \boldsymbol{Q}_{p} & \boldsymbol{Q}_{w}^{T} \boldsymbol{H} \boldsymbol{Q}_{w}
\end{array}\right] } \\
\boldsymbol{q}_{k}^{j}= & {\left[\begin{array}{c}
\overline{\boldsymbol{\sigma}}_{k}^{E}\left(t_{j}\right)^{T} \boldsymbol{H} \boldsymbol{\sigma}_{k 0}^{E} \\
\boldsymbol{Q}_{p}^{T} \boldsymbol{H} \boldsymbol{\sigma}_{k 0}^{E} \\
\boldsymbol{Q}_{w}^{T} \boldsymbol{H} \boldsymbol{\sigma}_{k 0}^{E}
\end{array}\right] } \\
d_{k}= & \frac{1}{2} \boldsymbol{\sigma}_{k 0}^{E}{ }^{T} \boldsymbol{H} \boldsymbol{\sigma}_{k 0}^{E}-\sigma_{y}{ }^{2}
\end{aligned}
$$

where $\boldsymbol{Q}_{p}$ and $\boldsymbol{Q}_{w}$ are the following matrices, computed at each integration point $k$

$$
\begin{aligned}
& \boldsymbol{Q}_{p}=\boldsymbol{E} \boldsymbol{B} \boldsymbol{A}_{p}-\boldsymbol{E} \\
& \boldsymbol{Q}_{w}=\boldsymbol{E} \boldsymbol{B} \boldsymbol{A}_{w}
\end{aligned}
$$

Using equation (35) and expressing constraint (II) of equation (32) by means of (25), (29) and (30) the final formulation of the quadratically constrained linear optimisation problem is

$$
\begin{aligned}
& \beta_{S}=\max _{\beta, \boldsymbol{w}, \boldsymbol{p}} \beta \quad \text { subject to: } \\
& \text { (I) } \frac{1}{2}\left[\beta \boldsymbol{p}^{T} \boldsymbol{w}^{T}\right] \boldsymbol{Q}_{k}^{j}\left[\beta \boldsymbol{p}^{T} \boldsymbol{w}^{T}\right]^{T}+\boldsymbol{q}_{k}^{j^{T}}\left[\beta \boldsymbol{p}^{T} \boldsymbol{w}^{T}\right]^{T} \leq-d_{k} \quad k=1, \ldots, K, \\
& j=1, \ldots, J \\
& \text { (II) } \beta \boldsymbol{M}-\boldsymbol{N}^{T} \boldsymbol{\kappa} \boldsymbol{C} \boldsymbol{K}^{-1} \boldsymbol{D}^{T} \boldsymbol{E} \boldsymbol{p}+\boldsymbol{\kappa} \boldsymbol{w} \leq-\boldsymbol{N}^{T} \boldsymbol{r}_{0}^{E} \\
& \text { (III) } \beta \geq 0 \\
& \text { (IV) } \boldsymbol{w}_{n}=\mathbf{0} \\
& \text { (V) } \boldsymbol{r}_{n}(\boldsymbol{w}, \boldsymbol{p}) \geq \mathbf{0}
\end{aligned}
$$

\section{Illustrative example}

In this paragraph we provide an illustrative example, consisting of a plate of unit thickness made of elastic-plastic material with yield stress $\sigma_{y}$ and containing a frictional crack with a constant coefficient of friction $\mu$ along the interfaces, as shown in Figure 3a. The dimensions are: plate width $2 b$, plate height $2 h$, crack length $2 a$. The distance $\delta$ indicates the vertical offset 
of the crack from the mid-plane of the plate. Notice that if the crack is centred $(\delta=0)$, the geometry and the loading is symmetric and the contact problem is uncoupled. By simply shifting the crack vertically, a condition of coupling can be enforced. Alternatively, coupling could also be enforced by considering a symmetric geometry where the crack lies along the interface between dissimilar materials or, alternatively, by considering a skewed crack. In the analyses we assumed $a / b=0.1, a / h=0.5, \delta / h=0$ and 0.25 .

The plate, under plane stress conditions, is loaded by a system of selfequilibrated tractions along the boundaries, whose normal and tangential resultants along the plate widths are indicated by $\boldsymbol{P}$ and $\boldsymbol{Q}$, respectively. The resultant $\boldsymbol{P}$ corresponds to a uniform compression pressing the crack faces, while the resultant $\boldsymbol{Q}$ is uniformly distributed along the four sides of the plate so as to produce an uniform shear within it. The following load path, expressed in terms of $\boldsymbol{P}$ and $\boldsymbol{Q}$, is considered, Figure $3 \mathrm{~b}$

$$
\boldsymbol{P}=\boldsymbol{P}_{0}+\beta \boldsymbol{P}_{0} \tan \gamma \cdot g(t), \quad \boldsymbol{Q}=\beta \boldsymbol{P}_{0} g(t)
$$

where $g(t) \in[0,1]$ is a general oscillating time function and $\gamma \in\left[0,45^{\circ}\right]$ is the angle defining the direction of the oscillating resultant acting along the plate widths. The load path of (39) is such that no separation can occur along the crack surface. If this condition is not satisfied, the system is likely not to shake down because a zone of microslip will almost always develop at the edge of the contact (Barber et al., 2008).

We discretised the model by means of the finite element method using 8 -node plane isoparametric elements with $2 \times 2$ integration points. Rigid body motion is prevented by appropriately constraining 3 dofs. The finite element model is comprised of 368 elements, with 1188 nodes, of which 35 are contact nodes. Thus, the size of the vector of plastic strains $\boldsymbol{p}$ is 4416 while that of the contact displacements $\boldsymbol{w}$ is 70 .

The illustrative example here considered is not aimed at any technical application but is believed to be suitable to show all the key features involved in frictional problems with plasticity. Other meaningful examples in the literature investigated the shakedown occurrence in specific mechanical components (see, for instance, the frictional slipping effects in pin-loaded lugs studied by Antoni, 2014 or the layered contact problem in Reina et al., 2011), though plasticity was not taken into account. 

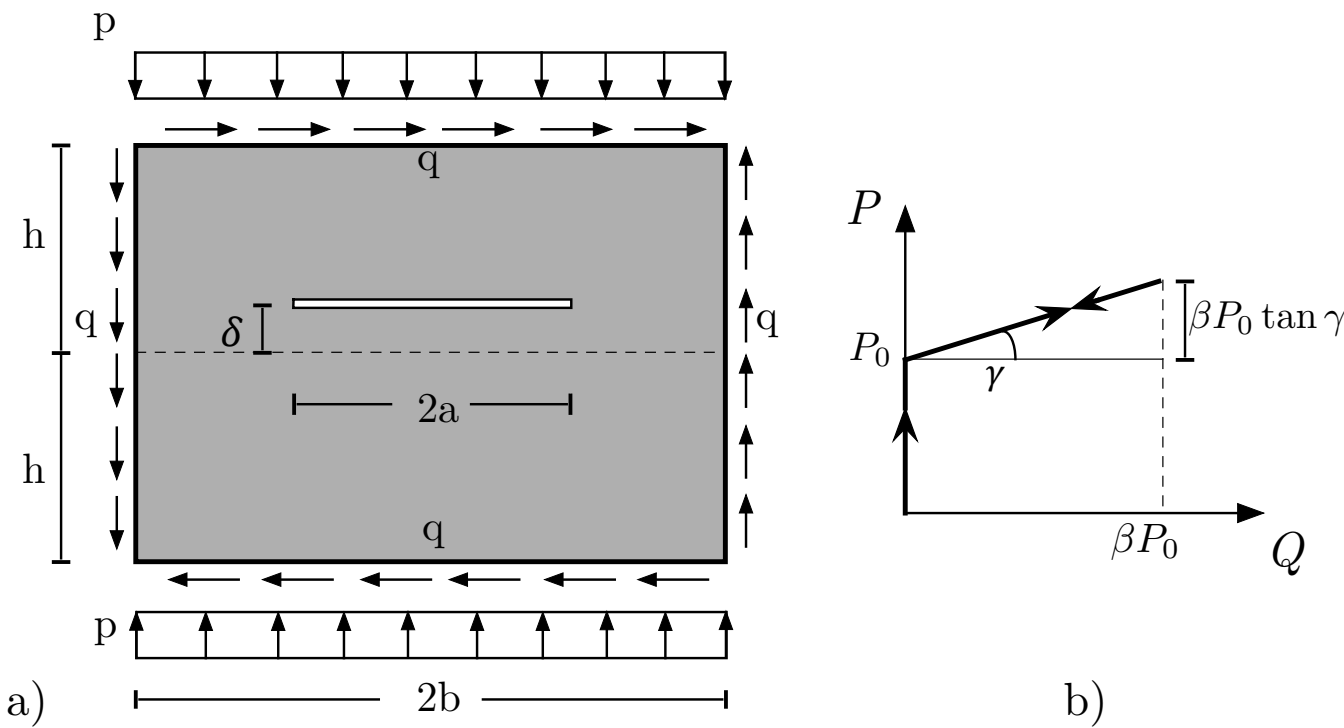

b)

Figure 3. a) Sketch of the model, where applied loads $p=P / 2 b$ and $q=Q / 2 b$ are depicted. b) Load path.

\subsection{Results}

We now present some of the most meaningful results obtained from the optimisation algorithms described in $\S 3$. All the matrices and vectors appearing in the formulation were computed on the discretised model described above and then implemented in the optimisation solver Gurobi 7.0 (Reference Manual, Gurobi Optimization Inc.) within Matlab environment. In order to facilitate the optimisation convergence, variables $\boldsymbol{p}$ and $\boldsymbol{w}$, as well as constraint (I) of equation (28) and constraints (I) and (II) of equation (38), were written in a normalized form. The results for the case of an elastic material are obtained from the linear optimisation described in $\S 3.2$ while in the case of elastic-plastic behaviour we used the quadratic optimisation of §3.3. It should be noted that the constraints in these two algorithms are different, therefore one cannot use the second algorithm on the elastic system as a limit case of the general elastic-plastic behaviour for $\sigma_{y} \rightarrow+\infty$. This is due to the fact that in algorithm (38) plastic strains are also part of the vector of variables and are involved in the linear constraint of the slip condition. In order to obtain comparable results with the linear optimisation of (28), one would have to force the plastic strain vector to be null. 

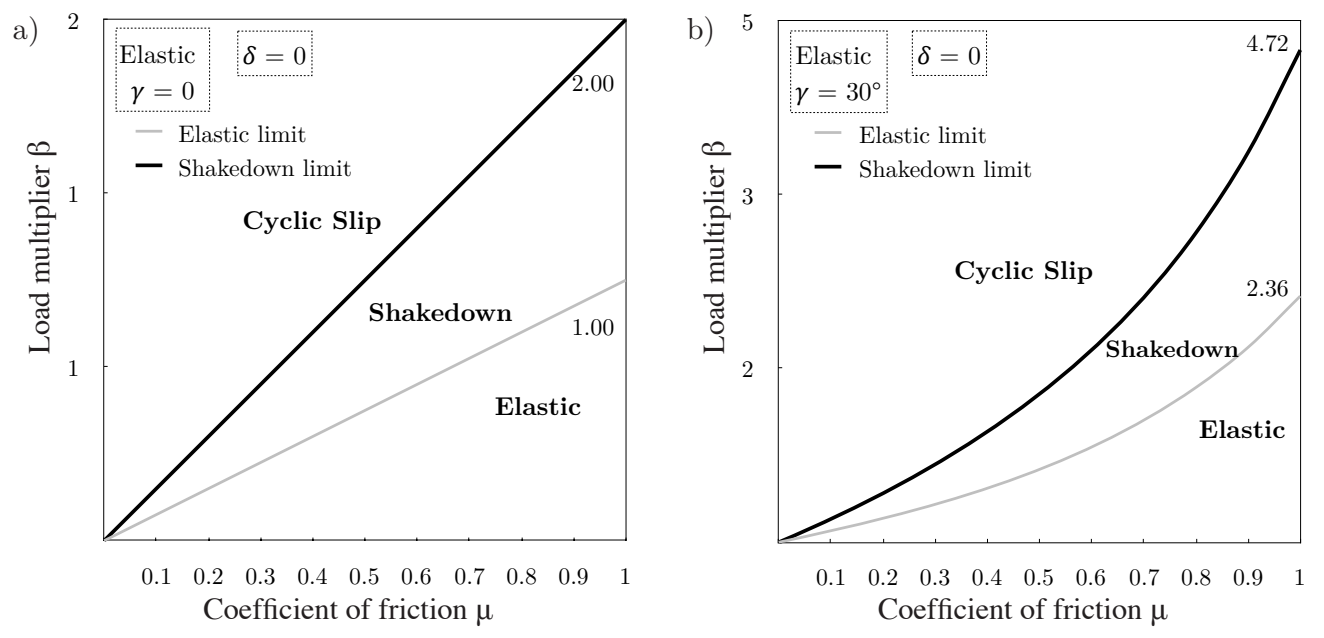

Figure 4. Uncoupled system $(\delta=0)$. State charts with elastic behaviour. (a) Load angle $\gamma=0^{\circ}$ and (b) load angle $\gamma=30^{\circ}$.

Firstly, the case of uncoupled contact (symmetric geometry, $\delta=0$ ) is considered. In Figure 4 the state chart of the elastic system is displayed, for two different values of the load angle $\gamma$. The lower lines mark the elastic limits, that is, the load level at which slip first occurs, i.e., constraint (I) in (28) is satisfied with $\boldsymbol{r}^{R}=\mathbf{0}$. The upper lines are the shakedown limits, which mark the maximum values of the load multiplier $\beta$ for which adaptation to an elastic response is possible. Above this level, the system behaviour is dissipative, namely it displays slip of opposite sign, a situation which is commonly known as cyclic slip. When $\gamma=0^{\circ}$, both profiles display a linear trend, and due to the uniformity and simplicity of the considered geometry and loads, the slope of the shakedown limit doubles that of the elastic limit. When $\gamma \neq 0^{\circ}$, the applied compressive stress is pulsating together with the shear stress and elastic and shakedown limits vary non-linearly with the coefficient of friction (see Figure 4b, where $\gamma=30^{\circ}$ ).

Figure 5 shows the optimal slip distributions for shakedown in dimensionless form, plotted against the normalized position along the contact interface $x / a$, for coefficients $\mu=0.5$ and $\gamma=0^{\circ}$. The dimensionless slip is defined as $\tilde{\boldsymbol{w}}_{\boldsymbol{t}}=\boldsymbol{w}_{\boldsymbol{t}} E /\left(a p_{0}\right)$, where $p_{0}=P_{0} / 2 b$ is the time-constant mean compressive stress applied to the plate. The lines show a parabolic profile, with the condition of zero slip at the tips of the crack and the maximum in the centre 


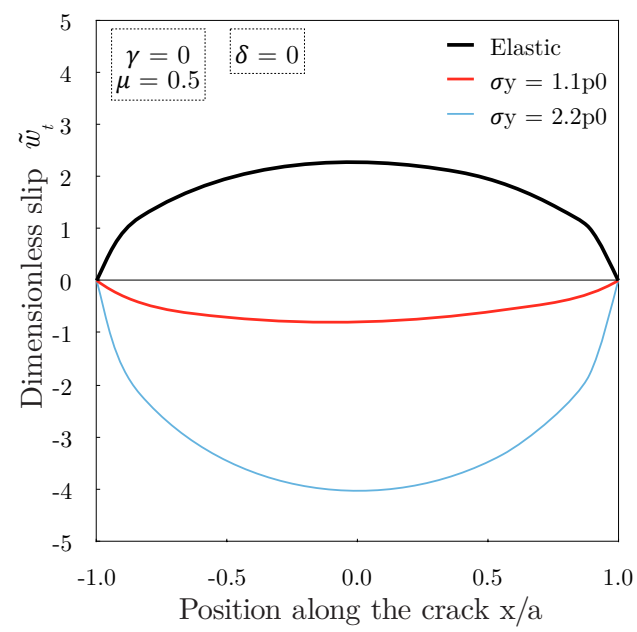

Figure 5. Uncoupled system $(\delta=0)$. Optimal residual slip distribution, for different values of the yield strength $\sigma_{y}$.

(the black line in the figure refers to the elastic case). (38)

In Figure 6 the trend of the shakedown limit is plotted against the coefficient of friction $\mu$ and the load angle $\gamma$. It can be noted that, as expected, increasing the coefficient of friction produces an increase of the shakedown limit. This increase is more pronounced for load angles different from zero. On the other hand, the effect of the load angle $\gamma$ is such that the shakedown limit increases with $\gamma$, particularly in the case of $\mu=0.8$ (dashed line in Figure 6b).

Now let us analyse the case with elastic-plastic behaviour. Figure 5 shows the optimal slip profile for shakedown, which displays the same parabolic shape of the elastic case, although the sign is opposite. This is due to the fact that in algorithm (38) plastic strains are also involved in the slip condition and their contribution is opposite to that of slips. Moreover, the absolute value of the maximum slip is now dependent on the value of the yield stress being considered.

In Figure 7 the state chart of the system is displayed, for two different values of the load angle $\gamma$. As in the previous case of the elastic system showed in Figure 4, the lower lines mark the elastic limits and the upper lines the shakedown limits. In this case, the elastic limit corresponds to the load level at which the constraints defined by the yield condition and the slip 

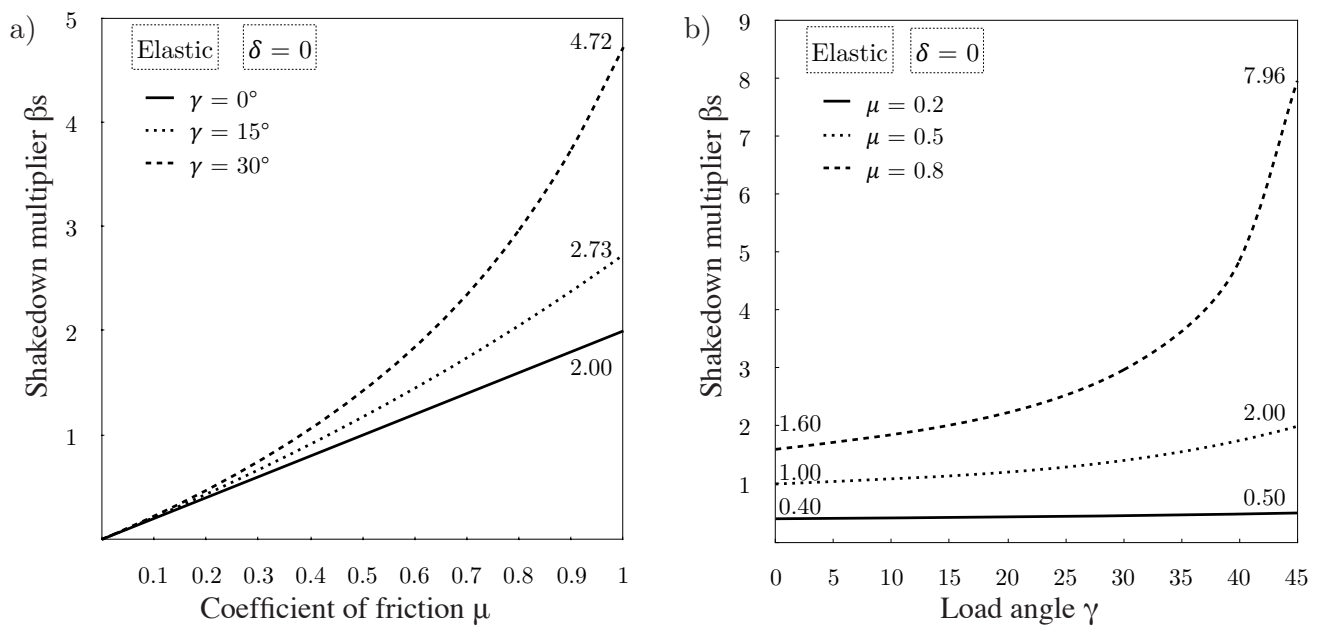

Figure 6. Uncoupled system $(\delta=0)$. Shakedown limit for elastic material. (a) Variation with coefficient of friction $\mu$. (b) Variation with load angle $\gamma$.

condition are satisfied, keeping the optimisation variables $\boldsymbol{w}$ and $\boldsymbol{p}$ null. It should be noticed that either elastic or shakedown limit conditions do not occur, generally, at the same time, meaning that possibly only one of the slip or yield constraints (I) and (II) in the algorithm (38) is satisfied as an equality. It can be observed that the elastic limit, as well as the shakedown limit, increases asymptotically with increasing friction (note that, due to the uniform distribution of elastic stresses within the plate, the asymptotic value of the elastic limit corresponds to the overall yielding of the plate itself).

In Figure 8 the trend of the shakedown limit is plotted against the coefficient of friction $\mu$ and the load angle $\gamma$. The case of an elastic material is compared with that of an elastic-plastic material for different values of the ratio $\sigma_{y} / p_{0}$. Two remarkable features can be noticed. On the one hand, Figure 8a, shakedown in the presence of an elastic-plastic material exhibits the asymptotic trend mentioned above for an increasing coefficient of friction $\mu$. It should be noticed that, for low values of the coefficient of friction, plastic deformation might create a residual stress field that helps to discourage frictional slip, namely a finite yield stress might make the system more likely to shake down. On the other hand, Figure 8b, the effect of plasticity might be such that the shakedown limit $\beta_{S}$ decreases with increasing load angle $\gamma$ regardless of the value of $\mu$, which is opposite to the trend recorded in the 


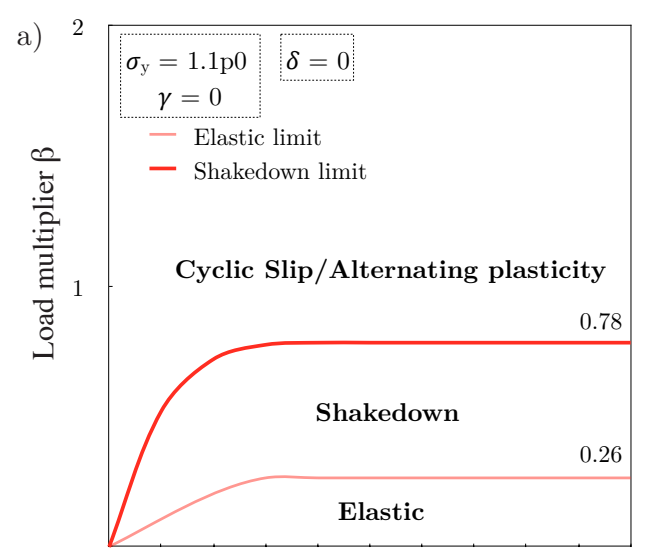

$\begin{array}{llllllllll}0.1 & 0.2 & 0.3 & 0.4 & 0.5 & 0.6 & 0.7 & 0.8 & 0.9 & 1\end{array}$ Coefficient of friction $\mu$

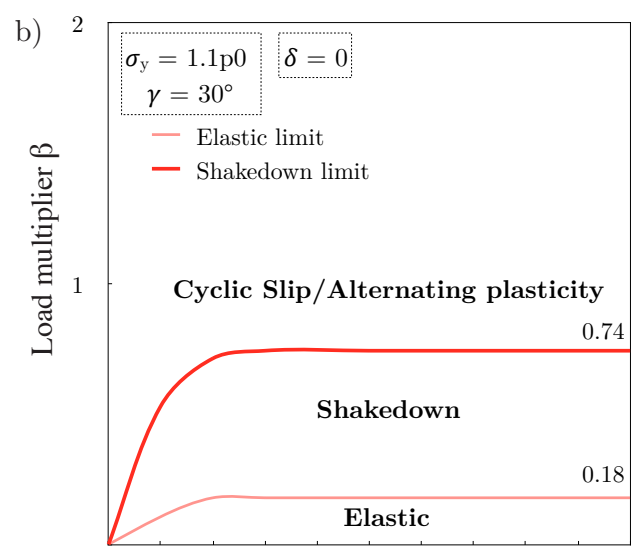

$\begin{array}{llllllllll}0.1 & 0.2 & 0.3 & 0.4 & 0.5 & 0.6 & 0.7 & 0.8 & 0.9 & 1\end{array}$ Coefficient of friction $\mu$

Figure 7. Uncoupled system $(\delta=0)$. State charts of the system with elasticplastic behaviour. (a) Load angle $\gamma=0^{\circ}$ and (b) load angle $\gamma=30^{\circ}$.

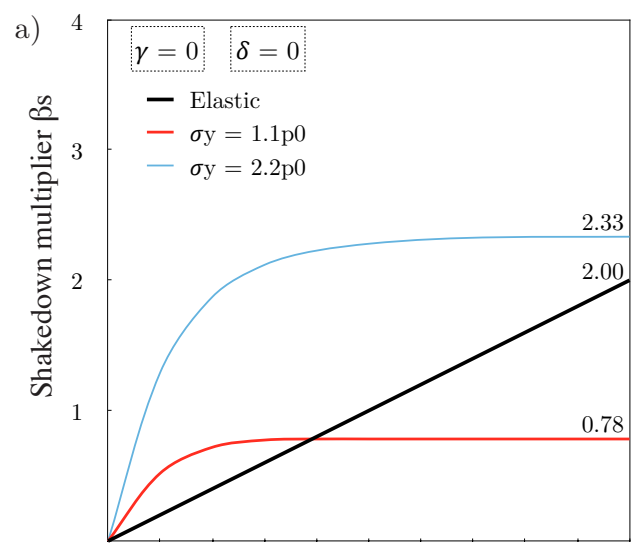

$\begin{array}{llllllllll}0.1 & 0.2 & 0.3 & 0.4 & 0.5 & 0.6 & 0.7 & 0.8 & 0.9 & 1\end{array}$ Coefficient of friction $\mu$

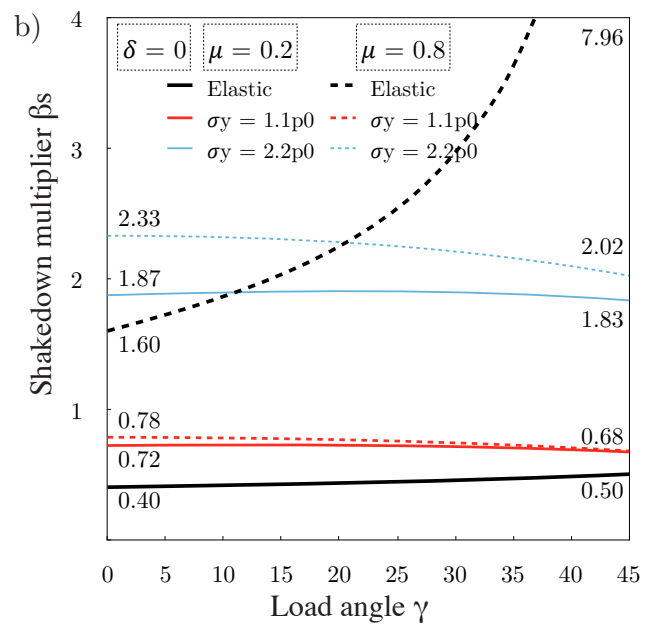

Figure 8. Uncoupled system $(\delta=0)$. Shakedown limit for elastic-plastic material. (a) Variation with coefficient of friction $\mu$. (b) Variation with load angle $\gamma$. 


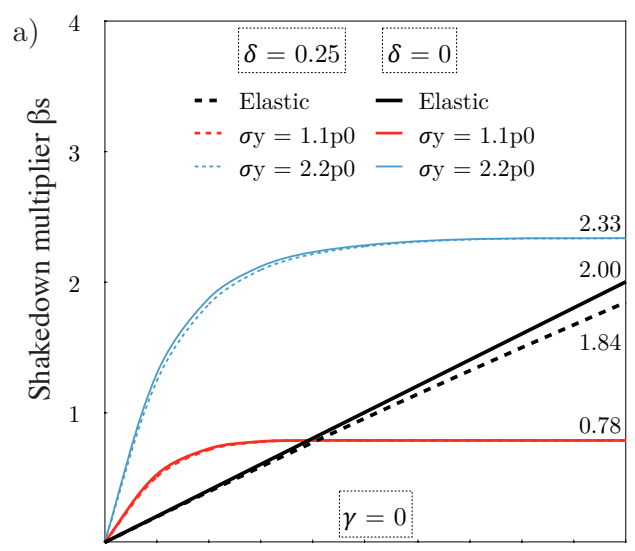

$\begin{array}{llllllllll}0.1 & 0.2 & 0.3 & 0.4 & 0.5 & 0.6 & 0.7 & 0.8 & 0.9 & 1\end{array}$ Coefficient of friction $\mu$

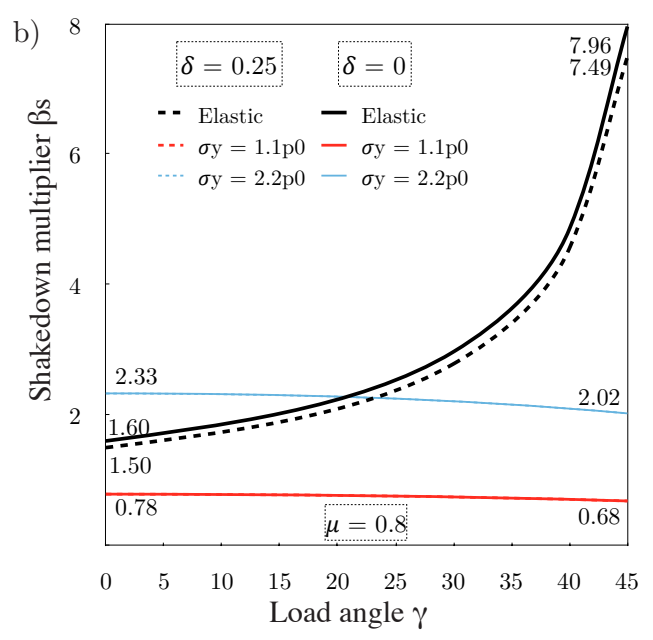

Figure 9. Shakedown limit for elastic-plastic material. Coupled $(\delta=0.25)$ and uncoupled system $(\delta=0)$ are compared. (a) Variation with coefficient of friction $\mu$. (b) Variation with load angle $\gamma$.

case of elastic material.

Finally, a case of coupled contact (asymmetric geometry, $\delta / h=0.25$ ) is considered. When the contact is coupled, the analogue of Melan's static theorem applied to frictional contacts provides only a necessary condition for shakedown, i.e., the existence of a residual state $\boldsymbol{r}^{R}$ in the elastic system or $\left(\boldsymbol{\sigma}^{R}, \boldsymbol{r}^{R}\right)$ in the elastic-plastic system, is no longer a sufficient condition for shakedown (Klarbring et al., 2007, 2017). Generally, for coupled contacts there exist (i) a load multiplier $\beta_{1}$ below which shakedown is guaranteed to occur and (ii) a load multiplier $\beta_{2}$ above which shakedown is impossible (Ahn et al., 2008). In this case, the parameter $\beta_{S}$ obtained from the optimisation problems (28) or (38) is the upper bound limit $\beta_{2}$, which means that shakedown is impossible for $\beta>\beta_{2}$ but for $\beta_{1}<\beta<\beta_{2}$ may or may not occur depending on initial conditions (Flicek et al., 2015).

\section{Conclusions}

The general shakedown problem of discrete systems involving friction and plasticity is presented. The formulation of the problem is discussed with reference to Coulomb's friction law and Von Mises' yield function, including 
elastic normal-tangential coupling at the contact surface and the interaction between friction and plasticity.

In Melan's static theorem (originally formulated in limit analysis of elasticplastic systems with associated plasticity), the existence of a time-independent residual state gives a sufficient condition for the system to shake down. A similar condition applies to elastic systems with frictional interfaces under study, with the fundamental limitation of normal-tangential uncoupling, in order to respect the associated nature of the flow rule.

In this paper, the shakedown limit for a given cyclic load path is calculated by means of a non-linear programming algorithm based on an extended version of Melan's theorem, where the variables involved in the optimisation are the kinematic quantities, slip at the interfaces and plastic strains in the bulk.

For illustrative purpose, a two-dimensional system consisting of an elasticplastic plate with a frictional crack, exposed to fluctuating compressive and shear loads, is discussed. The example is instrumental for showing the interactive trend of shakedown at the interfaces (corresponding to no energy dissipation due to frictional slip) and of shakedown in the bulk (corresponding to energy dissipation due to plastic strains) as coefficient of friction and yield strength are made to vary. The influence of the direction of the loading axis with respect to the crack plane is also discussed together with the effect of contact coupling.

To the authors' best knowledge, this work represents a first attempt to investigate in a fairly general manner the non-linear programming problem of shakedown in the presence of friction and plasticity.

\section{References}

Ahn, Y. J., 2015. Shakedown upper limit determination of coupled multinode discrete frictional systems to cyclic loading. J. Mech. Sci. Technol. 29 (2), 447-451.

Ahn, Y. J., Bertocchi, E., Barber, J. R., 2008. Shakedown of coupled twodimensional discrete frictional systems. J. Mech. Phys. Solids 56 (12), 3433-3440.

Andersen, E. D., Roos, C., Terlaky, T., 2003. On implementing a primal-dual interior-point method for conic quadratic optimization. Math. Program. 95 (2), 249-277. 
Antoni, N., 2014. A study of contact non-linearities in pin-loaded lugs: Separation, clearance and frictional slipping effects. Int. J. Non. Linear. Mech. 58, 258-282.

Barber, J. R., Klarbring, A., Ciavarella, M., 2008. Shakedown in frictional contact problems for the continuum. C. R. Mecanique 336 (1-2), 34-41.

Björkman, G., Klarbring, A., 1987. Shakedown and residual stresses in frictional systems. In: Gladwell, G.M.L., Ghonem, H., Kalousek, J. (Ed.), Contact Mechanics and Wear Rail/Wheel Systems II: Proc. 2nd Int. Symp. pp. 27-39.

Churchman, C. M., Hills, D. A., 2006. General results for complete contacts subject to oscillatory shear. J. Mech. Phys. Solids 54 (6), 1186-1205.

Churchman, C. M., Korsunsky, A., Hills, D. A., 2006. The Application of Plasticity Principles to Friction. J. Strain Anal. Eng. 41 (4), 323-328.

Corradi, L., Zavelani, A., 1974. A linear programming approach to shakedown analysis of structures. Comput. Method. Appl. M. 3 (1), 37-53.

Flicek, R. C., Hills, D. A., Barber, J. R., Dini, D., 2015. Determination of the shakedown limit for large, discrete frictional systems. Eur. J. Mech. A-Solid. 49, 242-250.

Gurobi Optimization Inc., 2016. Gurobi Optimizer Reference Manual (vers.7.0).

URL http://www.gurobi.com

Klarbring, A., Barber, J. R., Spagnoli, A., Terzano, M., 2017. Shakedown of discrete systems involving plasticity and friction. Eur. J. Mech. - A/Solids 64, 160-164.

Klarbring, A., Ciavarella, M., Barber, J. R., 2007. Shakedown in elastic contact problems with Coulomb friction. Int. J. Solids Struct. 44 (25-26), 8355-8365.

Koiter, W. T., 1960. General theorems for elastic-plastic solids. In: Progress in Solid Mechanics. Nort Holland, pp. 165-221.

König, J. A., 1987. Shakedown of Elastic-Plastic Structures. Fundamental Studies in Engineering. Elsevier Science, Amsterdam. 
König, J. A., Maier, G., 1981. Shakedown analysis of elastoplastic structures: A review of recent developments. Nucl. Eng. Des. 66 (1), 81-95.

Li, H. X., Yu, H. S., 2006. A nonlinear programming approach to kinematic shakedown analysis of frictional materials. Int. J. Solids Struct. 43 (21), 6594-6614.

Maier, G., 1969. Shakedown theory in perfect elastoplasticity with associated and nonassociated flow-laws: A finite element, linear programming approach. Meccanica 4 (3), 250-260.

Melan, E., 1936. Theorie statisch unbestimmter Systeme aus idealplastischem Baustoff. In: Akad. Wiss. Wien Sitzungsber. Vol. 145. pp. 195-218.

Michalowski, R. L., Mroz, Z., 1978. Associated and non-associated sliding rules in contact friction problems. Arch. Mech. 30 (3), 259-276.

Nowell, D., Dini, D., Hills, D. A., jan 2006. Recent developments in the understanding of fretting fatigue. Eng. Fract. Mech. 73 (2), 207-222.

Polizzotto, C., Borino, G., Fuschi, P., 2002. Shakedown of Structures Accounting for Damage Effects. In: Weichert, D., Maier, G. (Eds.), Inelast. Behav. Struct. under Var. Repeated Loads Direct Anal. Methods. Springer Vienna, Vienna, pp. 187-201.

Pycko, S., Maier, G., 1995. Shakedown theorems for some classes of nonassociative hardening elastic-plastic material models. Int. J. Plasticity 11 (4), 367-395.

Pycko, S., Mroz, Z., 1992. Alternative approach to shakedown as a solution of a min-max problem. Acta Mech. 93, 205-222.

Reina, S., Dini, D., Hills, D. A., Iida, Y., 2011. A quadratic programming formulation for the solution of layered elastic contact problems: Example applications and experimental validation. Eur. J. Mech. A/Solids 30 (3), 236-247.

Spagnoli, A., Carpinteri, A., Montanari, L., 2013. Application of the Shakedown Theory to Brittle-Matrix Fiber-Reinforced Cracked Composite Beams Under Combined Traction and Flexure. J. Appl. Mech. 81 (3), 031012-1-8. 
Telega, J. J., 1995. On Shakedown Theorems in the Presence of Signorini Conditions and Friction. In: Mróz, Z., Weichert, D., Dorosz, S. (Eds.), Inelast. Behav. Struct. under Var. Loads. Springer Netherlands, pp. 183201.

Weichert, D., 1986. On the influence of geometrical nonlinearities on the shakedown of elastic-plastic structures. Int. J. Plasticity 2 (2), 135-148. 